MITSUBISHI ELECTRIC RESEARCH LABORATORIES

http://www.merl.com

\title{
Non-Refractive Modulators for Encoding and Capturing Scene Appearance and Depth
}

\author{
Ashok Veeraraghavan, Amit Agrawal, Ramesh Raskar
}

TR2008-028 July 2008

\begin{abstract}
We analyze the modulation of a light field via non-refracting attenuators. In the most general case, any desired modulation can be achieved with attenuators having four degrees of freedom in ray-space. We motivate the discussion with a universal 4D ray modulator (ray-filter) which can attenuate the intensity of each ray independently. We describe operating of such a fantasy rayfilter in the context of altering the 4D light field incident on a 2D camera sensor. Ray-filters are difficult to realize in practice but we can achieve reversible encoding for light field capture using patterned attenuating mask. Two mask-based designs are analyzed in this framework. The first design closely mimics the angle-dependent ray-sorting possible with the ray filter. The second design [17] exploits frequency-domain modulation to achieve a more efficient encoding. We extend these designs for optimal sampling of light field by matching the modulation function to the specific shape of the band-limit frequency transform of light field. We also show how a hand-held version of an attenuator based light field camera can be built using a medium-format digital camera and an inexpensive mask.
\end{abstract}

CVPR 2008

This work may not be copied or reproduced in whole or in part for any commercial purpose. Permission to copy in whole or in part without payment of fee is granted for nonprofit educational and research purposes provided that all such whole or partial copies include the following: a notice that such copying is by permission of Mitsubishi Electric Research Laboratories, Inc.; an acknowledgment of the authors and individual contributions to the work; and all applicable portions of the copyright notice. Copying, reproduction, or republishing for any other purpose shall require a license with payment of fee to Mitsubishi Electric Research Laboratories, Inc. All rights reserved. 



\title{
Non-Refractive Modulators for Encoding and Capturing Scene Appearance and Depth
}

\author{
Ashok Veeraraghavan \\ University of Maryland \\ College Park, MD 20742 \\ vashokeumiacs. umd.edu
}

\author{
Amit Agrawal, Ramesh Raskar* \\ Mitsubishi Electric Research Labs (MERL) \\ Cambridge, MA 02139 \\ \{agrawal, raskar\}@merl.com
}

\author{
Ankit Mohan and Jack Tumblin \\ Northwestern University \\ Evanston, IL 60208 \\ \{mohan, jet\}@es.northwestern.edu
}

\begin{abstract}
We analyze the modulation of a light field via nonrefracting attenuators. In the most general case, any desired modulation can be achieved with attenuators having four degrees of freedom in ray-space. We motivate the discussion with a universal 4D ray modulator (ray-filter) which can attenuate the intensity of each ray independently. We describe operation of such a fantasy ray-filter in the context of altering the $4 D$ light field incident on a $2 D$ camera sensor.

Ray-filters are difficult to realize in practice but we can achieve reversible encoding for light field capture using patterned attenuating mask. Two mask-based designs are analyzed in this framework. The first design closely mimics the angle-dependent ray-sorting possible with the ray filter. The second design [17] exploits frequency-domain modulation to achieve a more efficient encoding. We extend these designs for optimal sampling of light field by matching the modulation function to the specific shape of the band-limit frequency transform of light field. We also show how a hand-held version of an attenuator based light field camera can be built using a medium-format digital camera and an inexpensive mask.
\end{abstract}

\section{Introduction}

Light fields characterize the irradiance of each ray in free space using a twin plane parameterization $[7,11]$. By capturing a light field of the scene, all the information content about the scene appearance can be obtained. We present a class of methods using non-refracting optical devices to capture the information content in the light field. The key

\footnotetext{
${ }^{*}$ Ramesh Raskar is currently with MIT Media Labs
}

idea is to individually attenuate each ray so that appropriate linear combinations measured by the sensor can be used to recover informative parts of the light field.

\subsection{Contributions}

Towards capture of scene information available in a light field, we make the following contributions.

- We describe a non-refractive ray-filter which can attenuate the intensity of each ray independently and support a rich array of imaging functionalities.

- We show the operation of this fantasy ray-filter in the context of altering the 4D light field inside a camera. We explain how the functionality of the ray-filter can be partially achieved using patterned attenuating masks and analyze two previously known camera designs in this framework.

- We extend the heterodyne light field camera design [17] to optimize for non-rectangular shape of the band-limited light fields and show how ray modulators can be designed for optimal sampling of the light field.

- We show an easy practical implementation of maskbased light field camera using a medium-format camera and inexpensive masks.

Our goal is not to invent new cameras but to analyze previously known designs using this generalized framework and to explore potential improvements based on the new understanding.

\subsection{Related Work}

Light Field Capture: Digital sensors are limited to be two dimensional surfaces while the light field is four dimensional. Therefore, it is necessary to modulate/transform it so 


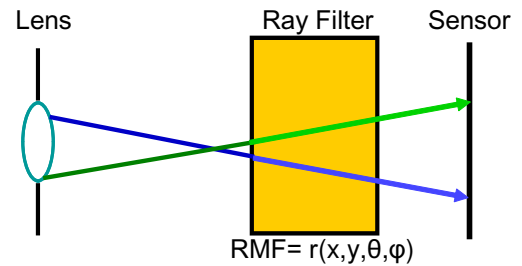

Figure 1. Conceptual design of a general ray modulator (ray-filter) with four degrees of freedom in ray space. The ray filter is capable of attenuating the intensity of each ray in 4D space independently.

that the information in the angular dimensions can be sampled by the sensor. A straightforward way to sample angular dimensions is viewpoint sampling. This can achieved by using a dense array of cameras, one for each viewpoint as in [18]. Such dense camera arrays, however, are impractical for consumer applications because of their sheer bulk.

Recently, two handheld light field cameras have been proposed by modifying a traditional camera using refractive elements. The first design uses an array of positive lenses with appropriate prisms in front of a main lens [6]. The second design (inspired by [1]) uses a microlens array in front of the sensor surface focusing the image of the main lens on the sensor [14]. These array of lenses or the microlens array rebin the incoming rays to enable sampling of light field using a 2D camera. However, all refractive modulators suffer from inherent limitations such as spherical/chromatic aberrations, coma and misalignment issues. Here, we analyze 'non-refractive' modulators which only attenuate light rays (instead of bending them) for light field capture.

Non-Refractive Light Field Modulators (NRLFM) such as masks have been used previously in context of coded aperture imaging, in astronomy [16] and computational photography $[10,17]$. Hiura \& Matsuyama [8] as well as Farid \& Simoncelli [5] describe two related methods for estimating depth from multiple images captured using coded apertures using defocus. Volume holograms [2] have been used for four dimensional spatio-spectral imaging. Zomet \& Nayar [19] proposed a volume of light attenuating layers that are controllable in space and time for a lensless imaging system and showed a variety of nonconventional imaging functionalities such as split field of view.

The use of patterned mask to recover light field from a single captured image was first proposed in [17]. In this paper, we take a broader look at the light fields and their modification based on a ray-filter. This is applicable to any band-limited light field with two plane parameterization including the light field inside a camera. We show that the heterodyne design of Veeraraghavan et al. [17] is a special case of this discussion.

In [17], the light field was assumed to be rectangularly bandlimited for simplicity. In practice, the frequency content of images and light fields decrease with increasing fre- quency. This fact can be utilized for optimal sampling of light fields. For example, quincunx sampling can reduce the sampling rate by a factor of two in digital video systems. Similarly, sampling circularly symmetric functions on a hexagonal lattice requires $13.4 \%$ fewer samples than rectangular sampling [9]. We show how to optimally sample incident light fields for non-rectangular shaped bandlimits, which leads to an increase in spatial resolution of the recovered light field over rectangular sampling.

\section{Light Field Modulation}

We first describe the concept of a ray filter for generic light field modulation.

\subsection{Ray-Filter: The Universal NRLFM}

An ideal ray modulator must possess four degrees of freedom so that each individual ray can be modulated independently. A thin sheet of some special material whose absorptiveness is precisely controllable both as a function of the spatial location and the direction of incoming ray, may be able to achieve such a modulation. We call this ideal modulator the 'ray-filter'. A ray-filter can be programmed to realize any possible non-refractive modulation and therefore is a 'universal non refractive modulator'. Figure 1 depicts a ray-filter in conjunction with a lens and a sensor showing that it attenuates each ray of the incoming light field independently.

However, we are unaware of any single optical element that can act as a ray-filter. Even the stack of light attenuating layers [19] may not be able to provide the required selectivity. Therefore, in practice, we are forced to settle for optical elements which have lower specificity. Nevertheless, one might be able to construct such a ray-filter in future using refractive optics: a color LCD filter (LCD display without backlight) with a lens array on both sides. For each lens, all rays arriving from a specific direction gets focussed at one point on the LCD filter, which selectively attenuates the RGB components. The outgoing rays then pass through the lens array on the other side which diverts them in a single direction.

\subsubsection{Ray Modulation Function}

The ray-filter can be mathematically characterized by Ray Modulation Function (RMF), $r(x, y, \theta, \phi)$. Since, the rayfilter does not bend the rays of light but rather attenuates it, the light field entering the ray-filter $(l)$ is related to the light field exiting the ray-filter $\left(l_{r}\right)$ as

$$
l_{r}(x, y, \theta, \phi)=r(x, y, \theta, \phi) l(x, y, \theta, \phi) .
$$

$r$ is constrained to be positive. Since multiplication corresponds to convolution in frequency domain, the effect of the 


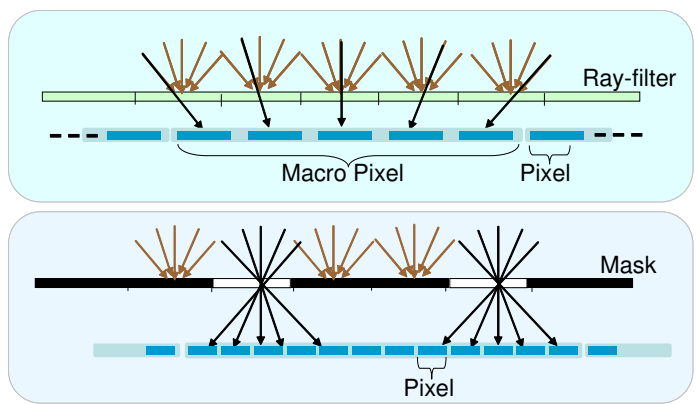

Figure 2. (Top) Ray-filter based light field camera. Within each macro pixel, the ray-filter allows only a single direction of rays to be sensed by a single pixel on the sensor, thus achieving angular sampling within the macro-pixel. (Bottom) A practical realization of the ray-filter using a pinhole array mask.

ray-filter can be written as

$L_{R}\left(f_{x}, f_{y}, f_{\theta}, f_{\phi}\right)=R\left(f_{x}, f_{y}, f_{\theta}, f_{\phi}\right) \otimes L\left(f_{x}, f_{y}, f_{\theta}, f_{\phi}\right)$,

where $\otimes$ denotes the convolution operator, and $L_{R}, R$ and $L$ denote the corresponding Fourier transforms. The image, $i$, formed on the 2D sensor is given by the integration of resulting light field over angular samples.

$$
i(x, y)=\int_{\theta} \int_{\phi} l_{r}(x, y, \theta, \phi) d \theta d \phi .
$$

From Fourier Slice theorem [13], the 2D image is also equivalent to a slice of 4D light field in Fourier domain.

$$
I\left(f_{x}, f_{y}\right)=L_{r}\left(f_{x}, f_{y}, 0,0\right),
$$

where $I\left(f_{x}, f_{y}\right)$ denote the Fourier transform of sensor image.

Notice that contrary to filtering, the effect of the ray-filter is a multiplication in the primal domain and a convolution in the Fourier domain. Depending on the nature of the information content in the light field, appropriate RMF's must be designed with corresponding algorithms for recovering this information from the captured sensor pixels. For example, selection of a particular viewpoint $(\theta, \phi)$ can be achieved by setting the ray-filter such that only the rays that come from that particular direction pass through. Next, we discuss optical devices that can be realized using such a ray-filter and specify their appropriate RMFs.

\subsection{Ray-filter Light Field Camera}

It is easy to design a light field camera using such a programmable ray-filter. Suppose we wish to capture a light field of spatial resolution $100 \times 100$ and angular resolution $5 \times 5$ using a camera with $500 \times 500$ pixels. This may be achieved by programming the ray-filter in blocks of $5 \times 5$ pixels. Within each array of $5 \times 5$ pixels, the ray-filter selects rays from one particular angle to reach a particular pixel

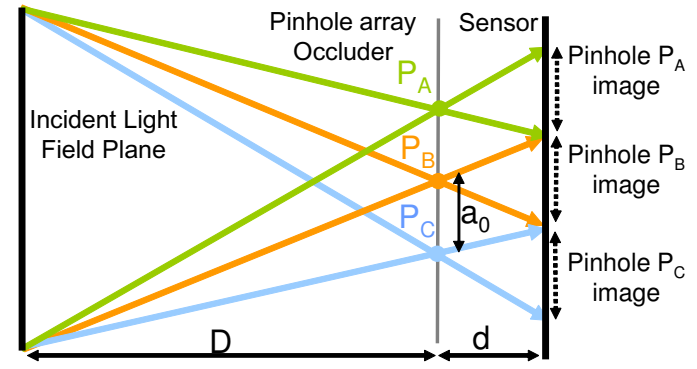

Figure 3. Schematic showing a non-refractive modulation based design for the micro lens array based light field camera [14]. Pinholes are separated by a distance $a_{0}$ and each pinhole is colored differently in the schematic for illustrative purposes. The ray modulation function for this design may be realized using a mask at the appropriate distance from the sensor.

while other rays are blocked. The problem with such a design is that most of the light reaching the sensor is blocked, and the SNR reduces drastically.

To increase the light efficiency, one could allow each sensor pixel to sample a linear combination of the angular light field samples of that particular macro-pixel. Rayfilter design now boils down to selection of invertible linear combinations (e.g. Hadamard coding) such that light field samples may be recovered from the sensor measurements by inverting those linear combinations.

\subsection{Practical Issues: Masks for Modulation}

Currently, optical devices having the specificity and control to act as a ray-filter are not available. However, it is fairly easy to print patterned masks which are 2D light attenuating patterns. These masks can be placed between the main lens and the sensor at any desired location and tilt. Masks can be printed at resolutions reaching as low as 25 nanometer with 1024 gray levels and their placement between the lens and the sensor can also be precisely controlled. Note that not all RMF's may be realized using patterned masks since masks offer different attenuation for every spatial location, but equally for all rays falling on that location. In the next section, we will analyze RMF's that can be realized using patterned masks for capturing light fields.

\section{Light Field Camera Designs}

In this section, we analyze several light field (LF) camera designs based on masks using ray filters. We show that heterodyne light field camera using cosine masks proposed in [17] is actually an extension of a pinhole-array mask LF camera. While the mask proposed in [17] assume bandlimited light fields with rectangular bandlimits, we show how to design masks for optimally sampling the light fields with non-rectangular bandlimits. 


\subsection{Pinhole Array based Light Field Camera}

$\mathrm{Ng}$ et al. [14] showed a hand-held light field camera by placing a microlens array in front of the sensor. The key idea was to sample the rays from a focused scene point by diverting them to different pixels using a micro-lens array, which otherwise would fall on a single pixel.

One can replace the microlens array with an array of pinholes and obtain the non-refractive analog of $\mathrm{Ng}$ 's camera as shown in Figure 3. This design (first proposed by Lippmann in [12]) can be realized using a ray-filter with the RMF given by

$$
r(x, y, \theta, \phi)= \begin{cases}1 & \text { if }(x, y)=\left(i a_{0}, j a_{0}\right) \quad(i, j) \in Z \\ 0 & \text { Otherwise },\end{cases}
$$

where, $a_{0}$, the separation between adjacent pinholes is such that images from adjacent pinholes do not overlap. If $A$ is the size of the aperture, $D$ is the distance between the lens and the pinholes and $d$ is the distance between the pinholes and the sensor, then the pinhole separation $a_{0}$ is given by $a_{0}=\frac{A d}{D+d}$ as shown in Figure 3. The image behind each individual pinhole captures a slightly different viewpoint thus capturing the light field.

Frequency Domain Interpretation: Since this RMF corresponds to an impulse train (5), in Fourier domain $R$ is also a series of impulses, as shown in Figure 4(a). Let the incoming light field be bandlimited to $f_{x 0}$ and $f_{\theta 0}$ as shown in Figure 4(a). If this light field is modulated by such a modulator, each of these impulses will create a spectral replica of the light field at the center of each impulse (Modulation Theorem [15]). The Figure 4(b) shows the continuum of copies of the light field corresponding to the series of impulses. The sensor image corresponds to the horizontal slice of the light field (shown in yellow). This design suffers severely from light-loss as any pinhole based implementation does. This can also be seen from the fact that though a continuum of spectral copies of the light field are created, only a very small subset of them is actually sensed by the sensor (Figure 4 ).

\subsection{Heterodyne Light Field Camera}

To alleviate the light-loss problem of a pinhole array, we need to achieve a more efficient light field modulation. First, we would like to move away from a pin-hole design to a mask which lets through more light. Second, in the frequency domain, we would like only a limited number of replicas depending on the frequency resolution in angular direction. In the example shown in Figure 5, we only need five impulses. In [17], this exact solution was proposed using the sum-of-cosines mask.

However, authors in [17] arrived at this solution from a very different direction, i.e., by comparing it with a lenslet

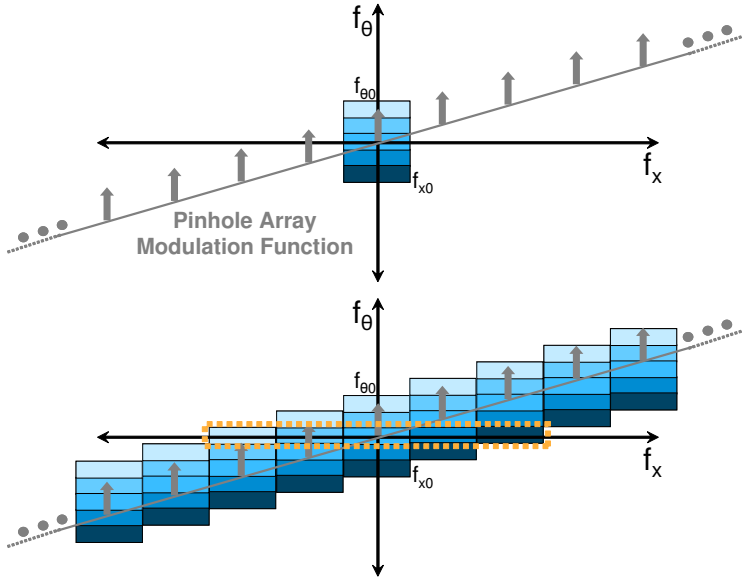

Figure 4. (Top) Bandlimited light field and the ray modulation function of an array of pinholes (an infinite train of impulses). (Bottom) The modulated light field consists of a train of (infinite) spectral copies of the light field. There is significant energy loss since several of these spectral copies cannot be sensed by the sensor slice shown by the yellow box.

based design. Our analysis shows that the sum-of-cosines mask based solution proposed in [17] is actually a special case of this generalized RMF which consist of impulses in the frequency domain. For completeness, let us look at the solution proposed in [17]. The RMF for this design can be described by

$$
R\left(f_{x}, f_{\theta},:\right)=\sum_{i=-p}^{i=p} \delta\left(f_{x}-2 i f_{x 0}, f_{\theta}-2 i f_{x 0} \tan (\alpha),:\right) .
$$

Here $\alpha$ is the angle along which the $(2 p+1)$ impulses are located and it depends on the distance between the mask and the sensor. Recovering the light field from the sensor image can be done in software by rearranging the 2D Fourier transform of the sensor image into $4 \mathrm{D}$ and taking the inverse 4D Fourier transform. (see [17] for more details).

\subsection{Optimal Sampling of Light Fields}

The design in [17] was optimized assuming that the shape of the band-limit was rectangular as shown in Figure 5. But in real-world scenarios, the incident light field spectrum has specific shape characteristics that are heavily dependent upon the depth of objects in the scene [3, 4]. We show how to optimize the mask so as to match the shape of the band-limit in the frequency domain. Usually, the spatial resolution of light field is reduced by a factor equal to the number of angular samples in captured light field. However, by optimizing the mask, better spatial resolution can be achieved as shown below. For illustration, we assume 2D light fields captured by 1D sensor, but it easily extends to 4D light fields captured by 2D sensor.

Let us assume that the band-limit light field is shaped 

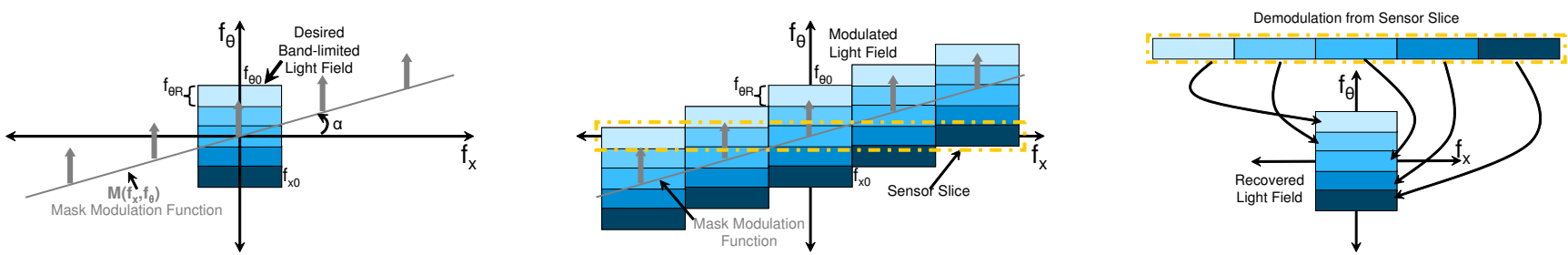

Figure 5. Spectral slicing in heterodyne light field camera for capturing 2D light field on 1D sensor. (Left) In Fourier domain, the sensor image is a slice of light field (horizontal axis, $\left(f_{\theta}=0\right)$ ). Without a mask, sensor can't capture the entire $2 \mathrm{D}$ light field spectrum (in blue). Mask spectrum (gray) forms an impulse train tilted by the angle $\alpha$. (Middle) By the modulation theorem, the sensor light field and mask spectra convolve to form spectral replicas, placing light field spectral slices along sensor's broad $f_{\theta}=0$ plane. (Right) To re-assemble the light field spectrum, translate segments of sensor spectra back to their original $f_{x}, f_{\theta}$ locations.
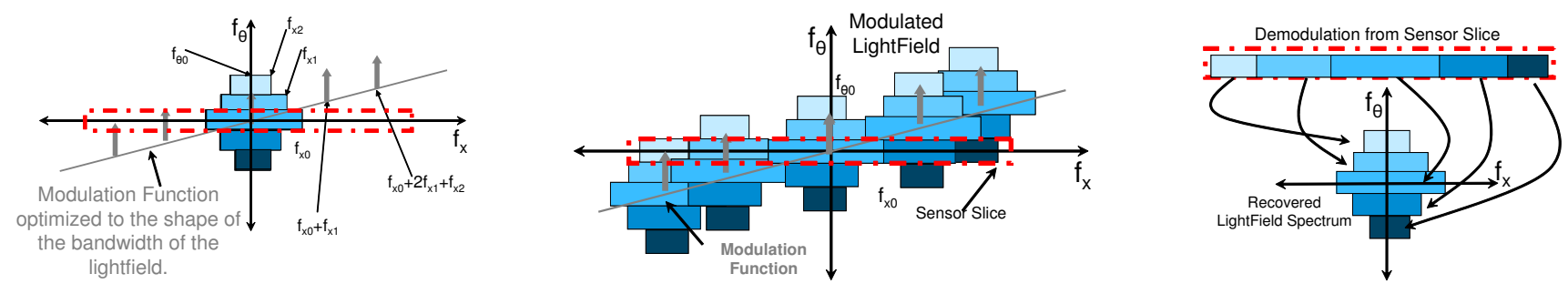

Figure 6. Optimal sampling of light fields. (Left) The bandlimit of the light field is not rectangular as in [17]. (Middle) The light field is modulated with cosines of appropriate frequencies (non-harmonics) so that the spectral replicas abut tightly on the sensor slice and there is no wastage of sensor pixels. Note that the spectral replicas could overlap in other parts of the spectrum which are not captured by the sensor. (Right) Demodulation involves reshaping the sensor Fourier transform as before accounting for unequal spectrum width in different angular samples.

as shown in Figure 6(a), with reducing spatial bandwidth as the angular frequency increases. Let the band-limits be given by $\left(f_{x 0}, f_{x 1}, f_{x 2}\right)$ corresponding to the angular frequencies $\left(f_{\theta}=0, f_{\theta}=f_{\theta R}, f_{\theta}=2 f_{\theta R}\right)$ as shown in Figure 6. Now consider a ray modulation function given by

$$
R\left(f_{x}, f_{\theta},:\right)=\sum_{i=-p}^{i=p} \delta\left(f_{x}-f_{i}, f_{\theta}-f_{i} \tan (\alpha),:\right),
$$

where $f_{i}=0, \quad i=0$ and $f_{i}=f_{x 0}+2 \sum_{j=1}^{j=i-1} f_{x j}+$ $f_{x_{i}}, \forall i>0$. This ray modulation function will lead to a series of unequally placed impulses and corresponding spectral copies of the light field as shown in Figure 6. The sensor image (red box) is a slice of the modulated light field (from Fourier Slice Theorem [13]). Note that the modulation function is now optimized so that the spectral copies are tightly abut on the sensor without any gaps. If we have used a mask with impulses equally placed, it would have resulted in gaps on sensor slice corresponding to no information in the light field. Moreover, if the depth range of the scene is known apriori, this leads to a specific shape of the light field band-limit [3, 4] and one can potentially use this information to optimally sample the light field.

\subsection{Mask based Realization of RMF}

As shown in [17], when the bandlimit of the light field is assumed to be rectangular, this modulation can be achieved by placing a sum-of-cosines mask at appropriate distance from the sensor. In this case, the frequencies of the cosines are harmonics of the fundamental frequency. However, for a mask optimized to the shape of the band-limit, the higher frequencies are not harmonics of the fundamental frequency but rather depend upon the shape of the band-limit.

Solving for the Light Field: To recover the 2D light field from the sensor image, we compute the Fourier transform of the sensor image, reshape the Fourier transform into $2 \mathrm{D}$ and compute the 2D inverse Fourier transform (Figure 5(c) \& 6(c)).

Comparison of Modulators: Figure 7 shows a comparison of the modulation range of the various modulators described in the previous subsections. While, ideally a rayfilter can modulate the entire light field spectrum, the effect of both pinhole arrays and heterodyning masks are restricted to be on a line (plane in 4D). The slope of this line (plane) depends on the distance of the mask from the sensor.

\section{Implementation}

We built a hand-held prototype of the mask based light field camera using Mamiya 645ZD medium format digital camera. The sensor size is $36 \times 48 \mathrm{~mm}$ with a resolution of $5344 \times 4008$ pixels and pixel size of $8.9 \mu$. The mask was simply dropped on the protective glass sheet covering the sensor. This leads to a mask-sensor distance of $1.2 \mathrm{~mm}$ 


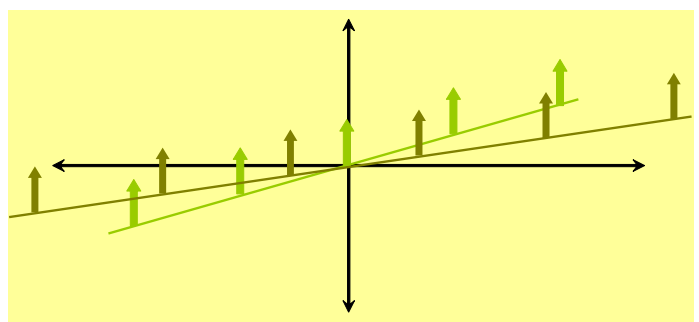

Figure 7. Modulation range of different ray filters in the frequency domain. (Yellow) The fantasy ray-filter can modulate any ray arbitrarily and hence occupies the complete band. (Brown) Pinhole arrays (parallel to sensor plane) can modulate only along a line in flatland (plane in 4D), with variable angle (depending on its distance from the sensor) and low magnitude spikes. (Green) The sum-of-cosines mask with the same resolution can modulate within the bandlimit of its highest cosine frequency with variable angle and higher magnitude spikes. The angle for pinhole array and heterodyning mask is shown different for the sake of clarity.

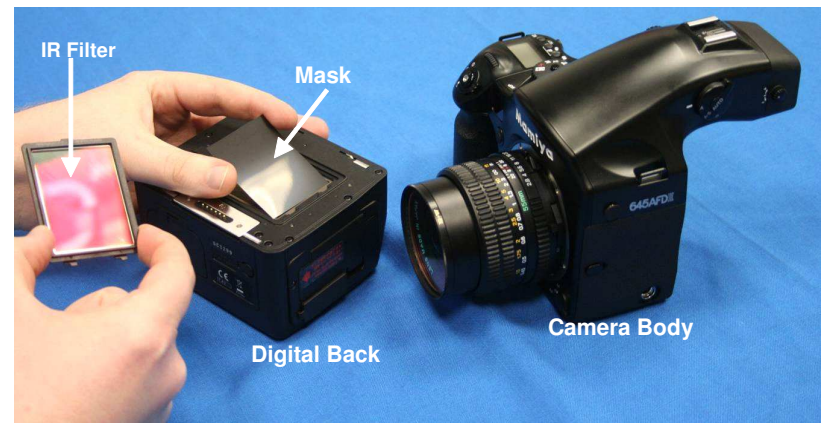

Figure 8. Hand-held digital light field camera. The mask is dropped on the protective glass on top of the sensor on the digital back. It is held in place using the IR filter that comes with the camera. The digital back is then attached to the camera body.

and restricts the maximum f-number that can be used to f/8. We used a $210 \mathrm{~mm}$ Mamiya Sekor lens. Figure 8(b) shows the medium format camera with the accessories used to implement the hand-held prototype. The mask is printed at 2032 DPI using a Kodak LVT (Light Valve Technology) film recorder on B\&W film from Bowhaus Inc. Four masks can be printed on a $4 \times 5$ inch $^{2}$ film for $\$ 50$. We printed a $2 \mathrm{D}$ sum of cosines mask with frequencies of $5,10,15,20$ cycles/mm allowing us to obtain $2 \times 4+1=9$ angular samples in the light field with spatial resolution of $240 \times 180$ (Results shown in Figure 9). We also printed another 2D sum of cosines mask with frequencies of $8,16,24$ cycles $/ \mathrm{mm}$ allowing us to obtain $2 \times 3+1=7$ angular samples in the light field with spatial resolution of $340 \times 250$ (Results shown in Figure 10).

\section{Applications}

\subsection{Depth from Focus}

Once we have captured the light field, images focused at any depth can be obtained by taking appropriate slices from the Fourier transform of the captured light field [13]. Figure 9(a) and 10(a) show the captured images of two scenes with significant texture and depth variations. Figures $9(b, c)$ and 10(b,c,d) show different refocused images for each dataset. We can extract depth from refocussed images since scene points that are not in focus are blurred while scene points in focus are sharp in the refocused images. So for a given pixel 'p', if we study a small region around the pixel, then, this region will be sharp in the refocused image at the correct depth while it will be blurred at all other depths. We use the variance of the neighborhood around each pixel as a measure of sharpness. Each pixel is assigned the depth corresponding to the refocused image in which the variance of its neighborhood is maximum. Such an assignment (10 depth levels) is shown in 9(d) and 10(e).

\subsection{All Focus Image}

We can also obtain the all-in-focus image just as is obtained in [13] from the estimated depth map and the corresponding refocussed images. For each pixel we choose the intensity from the refocused image corresponding to its estimated depth resulting in an all-in-focus images as shown in Figure 9(e) and Figure 10(f). Supplementary materials include Matlab code and input images as well as videos showing digital refocusing.

\subsection{D Texture mapped model}

We can also obtain a 3D texture mapped estimate of the scene, since we have the depth estimates and the corresponding irradiance(intensity) estimates. This allows us to recover a 3D texture mapped surface corresponding to the scene by appropriately combining the depth and irradiance estimates to create a 3D texture mapped surface. We can also synthesize novel views from the estimated 3D texture mapped model.

\section{Discussions}

Limitations: Any ray attenuation scheme leads to loss of light energy compared to refractive optics and consequently requires larger exposure time. This may lead to motion blur in outdoor scenarios. As ray attenuators become finer, they will introduce more diffraction artifacts. This might be a limiting factor in terms of the maximum resolution of light fields that may be sensed using such ray attenuators. For light field capture, it is assumed to be bandlimited. When this assumption is not satisfied, captured light fields may suffer from aliasing artifacts. We still have 
(a) Captured Modulated Image

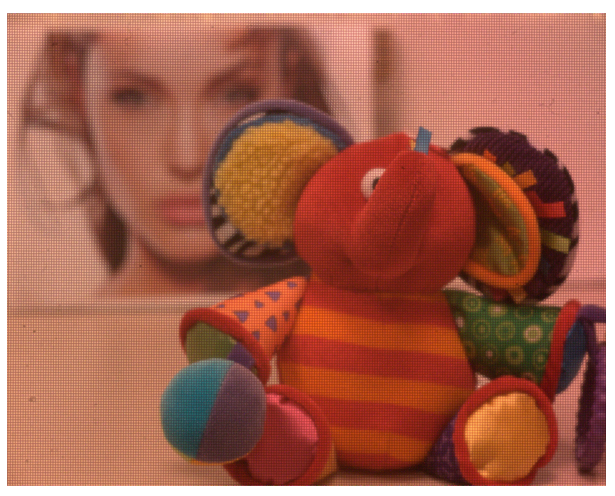

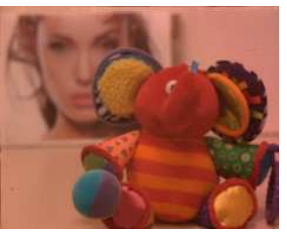

(b) Focus on Doll

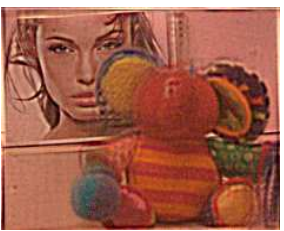

(c) Focus on face

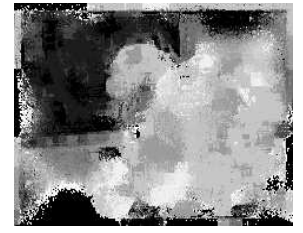

(d) Depth map

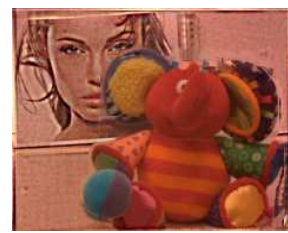

(e) All in Focus

Figure 9. (a) Captured Modulated Image (b) Refocussed Image-Focus in Front (c) Refocussed Image-Focus on the back end (d) Raw Depth labels quantized to 10 depth levels. (e) All in focus image.Supplemental material have additional results including refocussing videos and matlab code.

(a) Captured Modulated Image

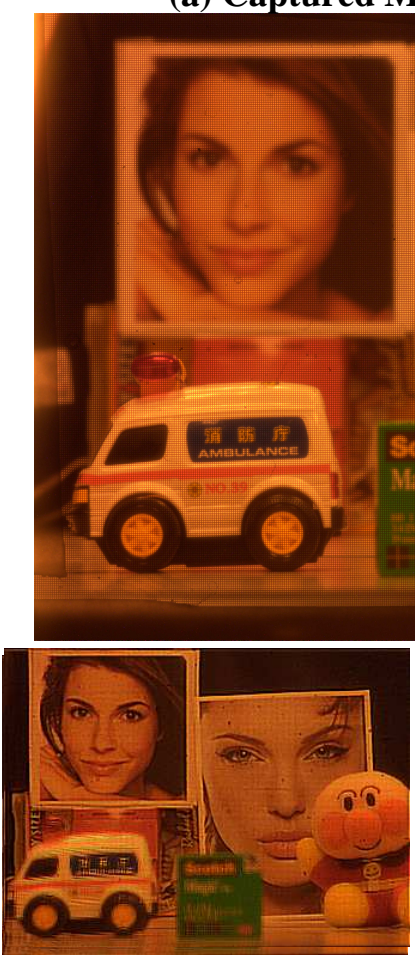

(b) Focus Back
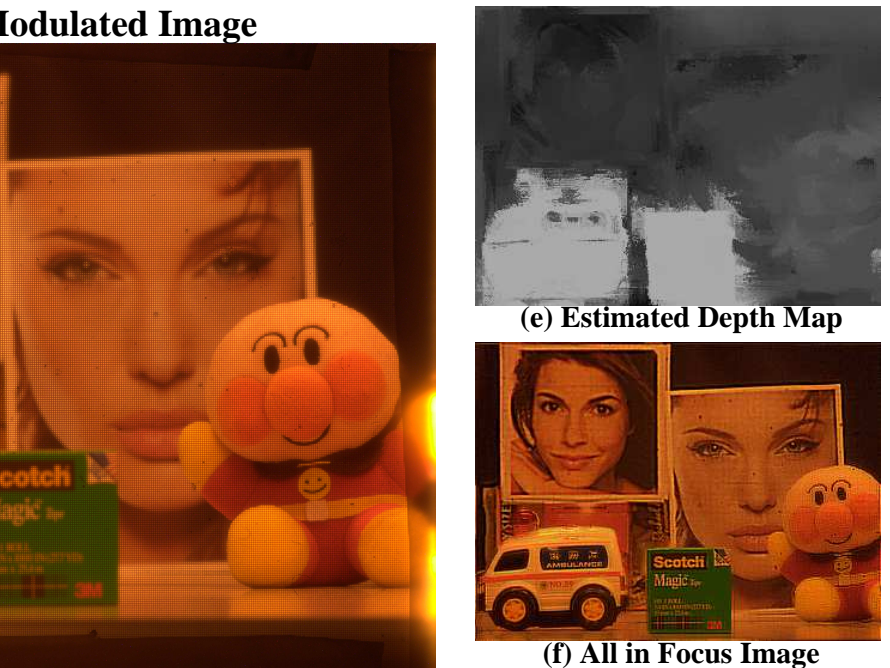

(e) Estimated Depth Map

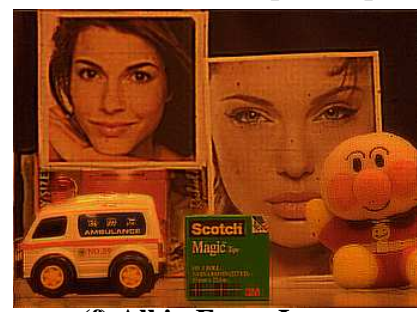

(f) All in Focus Image

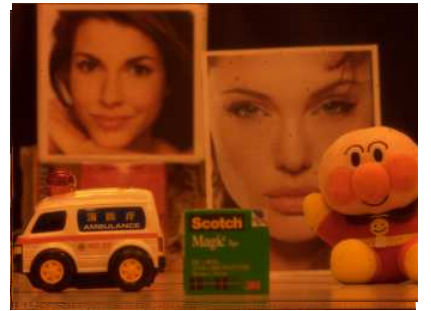

(c) Focus Center

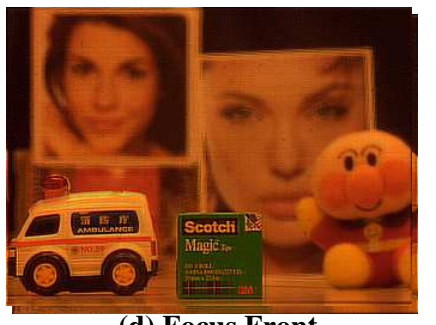

(d) Focus Front

Figure 10. (a) Captured Modulated Image using the Hand-held prototype(b) Refocussed Image-Focus on back poster (c) Refocussed ImageFocus on doll (d) Refocussed Image - Focus on front Scotch box (e) Raw Depth labels quantized to 10 depth levels. (e) All in focus image. Supplemental material have additional results including refocussing videos and matlab code.

not been able to design an optical pre-filter that can perform the required smoothening of the incoming light field in order to prevent aliasing. Since heterodyning requires linear combinations of light field samples, non-linearities such as saturation and specularities can lead to artifacts, if high dynamic range images are not captured.

Future Work: The range of imaging functionalities that may be obtained using a non-refracting attenuator may be significantly increased by considering ray-filters that are controllable both in wavelength and time. By using alternating ray-filters, one for capturing a high resolution image and another for capturing a band-limited light field we may be able to synthesize high resolution light fields. We can also use these high resolution light fields to obtain high resolution texture mapped 3D surface models. Another area of future research is designing highly selective and specific 
ray-filters using LCD screens and microlens arrays.

Conclusions: We described the theory of ray-filter based modulators and show that mask based modulation is an important special case of ray filters. We analyze the pinhole array and sum-of-cosines mask based light field capture designs in terms of ray filters. We extended the modulation based light field capture design to obtain optimal sampling of light fields for non-rectangular shape of the band-limits in the frequency domain.

\section{Acknowledgements}

We thank Rama Chellappa for useful comments, suggestions and support. We also thank Joseph Katz, Jay Thornton, John Barnwell, and other MERL members for their help and support.

\section{References}

[1] T. Adelson and J. Wang. Single lens stereo with a plenoptic camera. IEEE Trans. Pattern Anal. Machine Intell., 14:99106, 1992.

[2] G. Barbastathis and D. J. Brady. Multidimensional tomographic imaging using volume holography. Proc. IEEE, 87:2098-2120, 1999.

[3] J.-X. Chai, S.-C. Chan, H.-Y. Shum, and X. Tong. Plenoptic sampling. In SIGGRAPH, pages 307-318, 2000.

[4] F. Durand, N. Holzschuch, C. Soler, E. Chan, and F. X. Sillion. A frequency analysis of light transport. ACM Trans. Graph., 24(3):1115-1126, 2005.

[5] H. Farid and E. Simoncelli. Range estimation by optical differentiation. J. Opt. Soc. of America, 15(7):1777-1786, 1998.

[6] T. Georgiev, C. Zheng, S. Nayar, B. Curless, D. Salasin, and C. Intwala. Spatio-angular resolution trade-offs in integral photography. In EGSR, pages 263-272, 2006.

[7] S. Gortler, R. Grzeszczuk, R. Szeliski, and M. Cohen. The lumigraph. In SIGGRAPH, pages 43-54, 1996.

[8] S. Hiura and T. Matsuyama. Depth measurement by the multi-focus camera. In Proc. Conf. Computer Vision and Pattern Recognition, pages 953-961, 1998.

[9] A. Jain. Fundamentals of Digital Image Processing. Prentice-Hall, 1997.

[10] A. Levin, R. Fergus, F. Durand, and W. T. Freeman. Image and depth from a conventional camera with a coded aperture. ACM Trans. Graph., 26(3):70, 2007.

[11] M. Levoy and P. Hanrahan. Light field rendering. In SIGGRAPH, pages 31-42, 1996.

[12] G. Lippmann. La photographie integrale. CR Acad. Sci, 146:446-451, 1908.

[13] R. Ng. Fourier slice photography. ACM Trans. Graph., 24:735-744, 2005.

[14] R. Ng, M. Levoy, M. Brdif, G. Duval, M. Horowitz, and P. Hanrahan. Light field photography with a hand-held plenoptic camera. Technical report, Stanford Univ., 2005.
[15] A. V. Oppenheim, R. W. Schafer, and J. R. Buck. DiscreteTime Signal Processing. Prentice-Hall, 1999.

[16] G. K. Skinner. X-Ray Imaging with Coded Masks. Scientific American, 259:84, Aug. 1988.

[17] A. Veeraraghavan, R. Raskar, A. Agrawal, A. Mohan, and J. Tumblin. Dappled photography: Mask enhanced cameras for heterodyned light fields and coded aperture refocusing. ACM Trans. Graph., 26(3):69, 2007.

[18] B. Wilburn, N. Joshi, V. Vaish, E.-V. Talvala, E. Antunez, A. Barth, A. Adams, M. Horowitz, and M. Levoy. High performance imaging using large camera arrays. ACM Trans. Graph., 24(3):765-776, 2005.

[19] A. Zomet and S. Nayar. Lensless imaging with a controllable aperture. In Proc. Conf. Computer Vision and Pattern Recognition, pages 339-346, 2006. 